\title{
Overcoming the pitfalls of NGS-based molecular diagnosis of
}

\section{Shwachman-Diamond syndrome}

Xiaomin Peng ${ }^{1, *}$, Xinran Dong ${ }^{2, *}$, Yaqiong Wang ${ }^{2}$, Bingbing $\mathrm{Wu}^{2}$, Huijun $\mathrm{Wang}^{2,3}$, Wei $\mathrm{Lu}^{4}$, Feifan Xiao ${ }^{2}$, Lin Yang ${ }^{3}$, Gang $\mathrm{Li}^{2}$, Wenhao Zhou ${ }^{1,2,5}$, Bo Liu ${ }^{2,}{ }^{\dagger}$, Yulan $\mathrm{Lu}^{2,}{ }^{\dagger}$

\section{Affiliations}

1: Center for Molecular Medicine of Children's Hospital of Fudan University, Institutes of Biomedical Sciences, Fudan University, Shanghai, China

2: Molecular Medicine Center, Children's Hospital of Fudan University, Shanghai, China.

3: Shanghai Key Laboratory of Birth Defects, Pediatrics Research Institute,

Children's Hospital of Fudan University, Shanghai, China.

4: Department of Endocrinology and Inherited Metabolic Diseases, Children's Hospital of Fudan University, Shanghai, China.

5: Division of Neonatology, Children's Hospital of Fudan University, Shanghai, China.

*: These authors contributed equally to this work.

$\dagger$ : To whom correspondence should be addressed.

\section{Correspondence}

Bo Liu, Children's Hospital of Fudan University; 399 Wan Yuan Road, Shanghai, China,

201102; [boliu@fudan.edu.cn]; +86-21-64931016.

Yulan Lu, Children's Hospital of Fudan University; 399 Wan Yuan Road, Shanghai,

China, 201102; [yulanlu@fudan.edu.cn]; +86-21-64931016.

NOTE: This preprint reports new research that has not been certified by peer review and should not be used to guide clinical practice. 


\begin{abstract}
Purpose: Shwachman-Diamond syndrome (SDS) is predominately caused by biallelic mutations in the $S B D S$ gene and is characterized by exocrine pancreatic insufficiency, skeletal abnormalities and pancytopenia. Gene conversion between $S B D S$ and its pseudogene $S B D S P 1$ is the major cause. We established an efficient approach, HapICE, to infer the haplotype of $S B D S$ based on short-read next-generation sequencing (NGS). Methods: HapICE based on the Expectation-Maximization algorithm was developed to detect variants in exon 2 of $S B D S$ arising from gene conversion. We retrospectively analyzed two common pathogenic variants (c.183_184delinsCT and c.258+2T >C) in suspected SDS patients and compared the results with those from conventional NGS analysis.
\end{abstract}

Results: In 47 SDS high-risk individuals and 64 available parents, HapICE improved the diagnostic rate by $27.7 \%$ compared with conventional methods and revealed $100 \%$ (95\% CI: 92.5\%-100\%) concordance with Sanger sequencing. In addition to eighteen patients having consistent genetic results by both methods, HapICE further reported 8 patients with more accurate variant detection and 13 cases with the c.183_184delinsCT variant missing by conventional methods. HapICE also showed better performance in screening for carrier and wild-type status.

Conclusion: We have developed a novel $S B D S$ variant detection tool through regular NGS data that demonstrated precise variant detection performance in clinical scenarios.

\title{
1. Introduction
}

Short-read next-generation sequencing (NGS) has revolutionized the molecular diagnosis of genetic diseases and become the first-tier diagnostic assay in many clinical practices ${ }^{[1-4]}$. However, researchers and clinicians continue to face challenges due to high sequence similarity between homologous genes/regions and the accompanying gene conversion events. Gene conversion, a homologous recombination mechanism, often occurs between highly homologous parental genes/pseudogenes sharing a sequence similarity ranging from $92-99 \%{ }^{[5,6]}$. It is generally involved in the substitution of at least two neighboring but nonconsecutive markers within a short sequence $^{[5]}$. To date, abundant gene conversion events have been implicated as molecular causes and they play vital roles in human genetic disorders ${ }^{[7-10]}$. However, data analysis derived from short-read NGS is often hindered by pseudogene loci that are almost identical to orthologous loci and prevent the unique alignment of sequencing reads. Ambiguous read mapping will lead to inaccurate variant detection and concomitant misdiagnosis issues. Thus, how to accurately detect pathogenic variants resulting from gene conversion in homologous regions remains a challenge for NGS data analysis.

Shwachman-Diamond syndrome (SDS, MIM: 260400) is an autosomal recessive inherited disorder characterized by skeletal dysplasia, exocrine pancreatic dysfunction and pancytopenia ${ }^{[11]}$. It is the second most common cause of exocrine pancreatic 
insufficiency, with a reported incidence of 1 in 75,000 individuals ${ }^{[12]}$. Biallelic mutations in the Shwachman-Bodian-Diamond syndrome $(S B D S)$ gene are responsible for $90 \%$ of SDS patients ${ }^{[13-16]}$. Gene conversion between $S B D S$ and its unprocessed pseudogene $S B D S P 1$ results in two most common functional paralogous sequence variants (PSVs), c.183_184delinsCT and c.258+2T $>$ C, on exon $2^{[17]}$. These two functional PSVs account for approximately $80 \%$ of the $S B D S$ disease alleles ${ }^{[16,18-20] \text {, }}$ indicating the significance of accurate variant detection at these two loci in SDS molecular diagnosis.

Disentangling the disease-causing variants through short NGS reads is frequently limited since $S B D S$ shares $97 \%$ identity with $S B D S P 1{ }^{[21-23]}$. Yamada et al. reported two SDS patients with compound heterozygous mutations in the SBDS gene (c.258+2T $>\mathrm{C}$ on one allele and c.183_184delinsCT together with c.201A $>\mathrm{G}$ on the other), validated by Sanger sequencing. However, the c.183_184delinsCT and c. $201 \mathrm{~A}>\mathrm{G}$ variants were not identified by whole-exome sequencing (WES) in either patient due to mismapped reads, which pointed out the shortcomings of NGS data analysis for SDS diagnosis ${ }^{[22]}$. Since SDS usually presents with clinical heterogeneity and is thought to be underdiagnosed in the general population ${ }^{[12]}$, the omittance of pathogenic variants limited by ambiguous read mapping during conventional NGS analysis might be one of the possible causes.

Although the American College of Medical Genetics and Genomics (ACMG) guidance has pointed out that "laboratories must develop a strategy for detecting disease-causing variants within regions with known homology" [24], to our knowledge, few studies have confronted the analysis of variants arising from gene conversion based on short-read NGS data. In addition, although inaccurate molecular pathogenesis detection of the two most common $S B D S$ gene PSVs has been scatteredly reported ${ }^{[22]}$, a systematic description of the degree of error of standard analysis has seldom been assessed.

In this study, we developed an efficient computational approach named HapICE based on the Expectation-Maximization (EM) algorithm to infer the haplotype consisting of the PSVs for $S B D S$ molecular diagnosis and carrier screening through regular short-read NGS data. We retrospectively analyzed the two functional PSVs arising from $S B D S / S B D S P 1$ gene conversion among 47 suspected SDS patients by HapICE. The novel tool's analysis result was 100\% (95\% CI: 92.5\%-100\%) consistent with Sanger sequencing and showed an improved diagnostic rate of $27.7 \%$ compared with conventional NGS analysis, highlighting its advantages over standard NGS analysis for the detection of complex variants derived from gene conversion in clinical scenarios. We further compared the variant detection result among the enrolled 47 individuals and 64 available parents between two methods, finding that conventional NGS analysis resulted in $17.0 \%$ inaccurate pathogenesis diagnosis, $25.2 \%$ falsenegative, and $1.8 \%$ false-positive variant detections in the overall 111 individuals.

\section{Methods}

\subsection{Study design and data collection}


The outline of the study design is shown in Figure 1. The SDS high-risk cohort was retrospectively collected from deidentified individuals submitted to the Children's Hospital of Fudan University (CHFU) for genetic testing, with suggestions from clinicians and informed consent from parents. The inclusion criteria were patients who were 1) carriers of $S B D S$ variants of the two functional PSVs (c.183_184delinsCT and c. $258+2 \mathrm{~T}>\mathrm{C}$ ) or 2) clinically diagnosed as SDS, and conventional NGS analysis detected one heterozygous variant of either functional PSV. The detailed clinical inclusion criteria included 1) hematologic abnormalities characterized by persistent or intermittent neutropenia and 2) liver dysfunction with persistently elevated serum aminotransferase. Patients were excluded if 1) they were genetically diagnosed by pathogenic/likely pathogenic (P/LP) SNVs/small indels on other exons of SBDS or other established SDS-related genes or 2) they had severe infections, chronic viral hepatitis, toxicity and drug-induced liver injury, autoimmune diseases, or hemophagocytic syndrome. The detailed sequencing information is described in the Supplementary Notes.

\subsection{Conventional NGS variant calling of SNV/small indels}

Variant calling of $\mathrm{SNV} / \mathrm{small}$ indels from NGS data for conventional genetic diagnosis was performed following the pipeline described in our previously published studies $^{[1,25]}$. In brief, the sequencing reads were aligned to the GRCh37 (hg19) reference human genome using the Burrows-Wheeler Aligner (0.7.15-r1140 $)^{[26]}$. SAMtools (v.1.8) ${ }^{[27]}$ and Picard tools (v.2.20.1, http://broadinstitute.github.io/picard/) were used to sort, merge, and remove duplicated BAM files. SNVs and small indels were detected using the GATK tool and Best Practice Pipelines with default parameters (https://software.broadinstitute.org/gatk/best-practices).

\subsection{Haplotype inference for pseudogene-mediated conversion events based on short-read NGS data}

A computational approach, HapICE (Haplotype Inference for pseudogene-mediated Conversion Events), was established to detect variants created from SBDS/SBDSP1 gene conversion through short sequencing reads. The four main steps include:

\section{(1) Prepare the gene-specific combined reference:}

To identify the consistent and informative regions of the SBDS/SBDSP1 genes, the combined reference and annotation files were generated by the following steps: (i). Generate a combined reference file by $S B D S / S B D S P 1$ gene alignment with MUSCLE ${ }^{[28]}$ (v3.8.31), in which inconsistent bases between two genes are kept as origins and other consistent bases are marked as "N"; (ii). Generate a transition file that transferred the position on the combined reference to the original genomic position of the $S B D S / S B D S P 1$ genes; (iii). Generate the region annotation file in which the combined reference was classified into the target regions (different bases) and the other regions (consistent bases).

\section{(2) Generate the reads-to-region mapping content dataset:}

To collect the relevant reads that support the inference of potential haplotypes, reads from the BAM file were realigned to the generated combined reference sequence. First, 
reads that mapped to any position of the $S B D S / S B D S P 1$ genes were extracted by SAMtools ${ }^{[29]}$. Then, the extracted reads were remapped to the combined reference by BLAT ${ }^{[30]}$. Finally, the remapping result was transferred to a "reads-to-region mapping content" dataset, in which each row presented an extracted read, each column presented a target region, and each element recorded the exact base information at a given genomic region supported by a specific sequencing read.

(3) Parental gene/pseudogene haplotype inference:

\section{I: Problem statement for haplotype inference}

A categorical distribution was used to describe the haplotype information. Assuming there are $\mathrm{M}$ possible haplotypes $\left\{h_{1}, h_{2}, \ldots, h_{M}\right\}$ covering $\mathrm{K}$ target regions, the corresponding probability for haplotypes is $\theta=\left\{\theta_{1}, \theta_{2}, \ldots, \theta_{M}\right\}$, with $\sum_{m=1}^{M} \theta_{m}=1$. The categorical distribution is recorded as $\left\{\left(h_{m}, \theta_{m}\right)\right\}_{m=1}^{M}$. The goal of the algorithm is to estimate the haplotype probability $\theta$.

\section{II: Haplotype inference by Expectation-Maximization}

The EM algorithm ${ }^{[31]}$ was used to estimate the probability parameters $\left\{\theta_{M}\right\}$ for haplotype $\left\{h_{M}\right\}$ with sequencing reads $x_{n}$ as the observations. We use $\delta(x, h)$ to indicate whether a certain read $x$ can support haplotype $h$ (at least two intersecting target regions had no conflict). For the total $\mathrm{M}$ haplotypes and $\mathrm{N}$ reads, a binary matrix $D_{n * m}$ with ensembles $\left\{\delta_{n m}\right\}$ was used to denote whether read $n$ supported haplotype $m$.

\section{Algorithm deduction:}

\section{The E-step:}

Suppose the prior distribution for $h \mid \theta$ is the categorical distribution $\left\{\left(h_{m}, \theta_{m}\right)\right\}_{m=1}^{M}$. It can be derived that the posterior distribution for $h \mid x, \theta$ is $\left\{\left(h_{m}, \theta_{m}(x)\right)\right\}_{m=1}^{M}$, where

$$
\theta_{m}(x)=\frac{\delta\left(x, h_{m}\right) \theta_{m}}{\sum_{m} \delta\left(x, h_{m}\right) \theta_{m}}
$$

Given parameters $\theta^{(t)}$ at step $\mathrm{t}$ and sequencing read data $\left\{x_{n}\right\}$, the conditional expected log-likelihood Q-function is:

$$
\begin{gathered}
Q\left(\theta \mid \theta^{(t)}\right)=E_{h \mid x, \theta^{(t)}} \log p(h \mid \theta) \\
=\sum_{n} \sum_{m} \theta_{m}^{(t)}\left(x_{n}\right) \log \theta_{m}
\end{gathered}
$$

The M-step:

Maximizing the Q-function

$$
\theta^{(t+1)}=\arg \max _{\theta} Q\left(\theta \mid \theta^{(t)}\right)
$$

yields

$$
\begin{gathered}
\theta_{m}^{(t+1)} \propto \sum_{n} \theta_{m}^{(t)}\left(x_{n}\right) \\
=\sum_{n} \frac{\delta_{n m} \theta_{m}^{(t)}}{\sum_{m} \delta_{n m} \theta_{m}^{(t)}}
\end{gathered}
$$

\section{Algorithm implementation:}




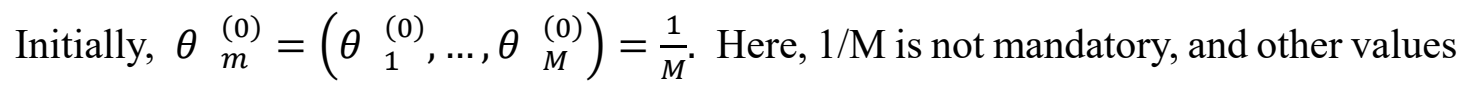
also work.

At each time t,

Calculate the posterior distribution matrix $P \underset{n m}{\stackrel{(t)}{n m}=\frac{\delta_{n m} \theta_{m}^{(t)}}{\sum_{m} \delta_{n m} \theta_{m}^{(t)}}}$

Sum according to haplotypes $\sum_{n} P \stackrel{(t)}{n m}$ and normalize to obtain $\theta \underset{m}{(t+1)}$

The iteration step will stop if convergence occurs $\sum_{m} \operatorname{abs}\left(\theta \stackrel{(t+1)}{m}-\theta_{m}^{t}\right)<10^{-10}$.

III: Reduce calculation complexity by a pruning and recursive strategy

Problem statement: each region $T_{k}$ contains the collection set of all possible sequence contexts in this region. Theoretically, the haplotype's state space is the Cartesian production of contexts of the K regions. Nevertheless, the probability values for most of the possible haplotypes are zero. Thus, we applied a recursive pruning strategy to reduce the calculation complexity.

Algorithm implementation:

For $\mathrm{k}=1$, directly perform the trivial estimation.

For $\mathrm{k}>1$, augment the state space from $\mathrm{k}-1$ to $\mathrm{k}$, i.e., haplotypes for $\mathrm{k}$ is the sequence concatenated from all remaining haplotypes from $\mathrm{k}-1$ with all possible sequence context at region $\mathrm{k}$. Do the EM step first and only reserve haplotypes with the highest probabilities (e.g., top 3 haplotypes) or only reserve haplotypes with probabilities higher than 0.01 .

Output the final haplotypes with the corresponding probability when $\mathrm{k}=\mathrm{K}$.

\section{(4): Result visualization:}

The proportion of gene recombination events between two neighboring informative bases, haplotype supportive read information, and the final inferred haplotypes of the parental gene/pseudogene pairs are displayed.

\subsection{Sanger validation}

Sanger sequencing was adopted to unambiguously study the mutational profile of $S B D S$ and $S B D S P 1$ using a well-established protocol developed in our laboratory. The protocol was based on long-range PCR amplification with $S B D S$ exon 2 allele-specific primers (forward: 5'-CTGCACCCCACCCCACCC-3', reverse: 5'TAAAAAATGAGTAACTGGATGGAG-3'), followed by DNA sequencing of smaller fragments (forward: 5'-AAAGAAAACTGCCCTCTACAC-3', reverse: 5'TCACATTATTGCTTGGTTAGTC-3').

\section{Results}

\subsection{NGS sequence alignment at $S B D S / S B D S P 1$ and possible interferences when} analyzing gene conversion events

Exon 2 of $S B D S$ and $S B D S P 1$ differed by only seven bases, and the relatively short sequencing reads frequently fell into homologous pitfalls from ambiguous alignment, 
resulting in incorrect variant calling, especially when variants arisen from gene conversion events (Figure 2). For example, we confronted a patient (Case 3) with compound heterozygotes of two pathogenic $S B D S$ alleles (the c. $258+2 \mathrm{~T}>\mathrm{C}$ allele and another allele comprised of c.183_184delinsCT and c.201A $>$ G) determined by trio Sanger sequencing. However, only the c. $258+2 \mathrm{~T}>\mathrm{C}$ variant was correctly detected by conventional NGS analysis (Figure 2B). A manual review found that nearly all of the NGS reads containing the two $S B D S$ variants, c.183_184delinsCT and c.201A $>$ G, were incorrectly aligned to the $S B D S P 1$ gene and mistakenly regarded as wild-type reads derived from $S B D S P 1$. At the same time, n.424 and n.533+10 of the $S B D S P 1$ gene were both mistakenly called heterozygous variants (Figure 2B and Figure 2C). The ambiguous mapping resulted in false-negative variant callings for $S B D S$ and falsepositive callings for SBDSP1 in regular NGS data analysis.

\subsection{Haplotype inference for SBDS/SBDSP1 gene conversion based on NGS data}

To solve the problem of inaccurate variant detection caused by the interference of highly homologous sequences, we developed an automatic tool, HapICE, to detect variants arising from gene conversion. We took the SBDS c.183_184delinsCT and c. $258+2 \mathrm{~T}>\mathrm{C}$ variants that were generated from $S B D S / S B D S P 1$ gene conversion as an application example (Figure 3).

Generally, since the read-mapping result in homologous regions is largely determined by PSVs, the PSV loci between $S B D S$ and $S B D S P 1$ can be used as anchor points to guide the short read mapping process. By inferring the haplotype block of these PSVs and calculating their corresponding proportions, HapICE can detect the variants that arise from gene conversion events and help make molecular diagnoses. Specifically, we aligned the genomic sequence of the $S B D S / S B D S P 1$ gene and generated a new combined reference to identify the consistent and informative regions of the genes. Then, we realigned the sequencing reads (from FastQ or BAM) to the combined reference sequence to collect supportive reads for the potential haplotypes of PSVs. Next, we performed EM algorithm by considering the haplotype composed of PSVs as the latent variable to infer the conversion haplotypes and reduce the calculation complexity by a pruning and recursive strategy. The haplotype inference result was eventually visualized in multiple aspects (Figure 3). The HapICE package is open source and available online at https://github.com/SherryDong/HapICE.

\subsection{Retrospective analysis of the SDS high-risk cohort}

After novel tool construction, we performed a retrospective analysis of c.183_184 and c.258+2 loci of the $S B D S$ gene in an SDS high-risk cohort. Altogether, 47 individuals from 46 unrelated families met the inclusion criteria, including 29 boys and 18 girls aged from newborn to 6 years old. Fourteen and 33 patients underwent clinicalexome sequencing (CES) and WES, respectively, with an average sequencing coverage of 199X and 103X.

Altogether, the HapICE reanalysis results showed that $39(83.0 \%)$ patients obtained diagnosable $S B D S$ haplotypes, and the other $6(12.8 \%)$ and $2(4.3 \%)$ individuals were carriers and wild-type at these two functional PSV loci, respectively (Supplementary 
Table 1). For the 39 patients with diagnosable $S B D S$ haplotypes, 31 (79.5\%) samples had both heterozygous variants, $2(5.1 \%)$ harbored a homozygous variant of c. $258+2 \mathrm{~T}>\mathrm{C}$, and the other $6(15.4 \%)$ had an allele of c.258+2T $>\mathrm{C}$ together with another allele of c.[183_184delinsCT;258+2T>C]. We performed Sanger sequencing on all of the enrolled patients and found that HapICE achieved 100\% (95\% CI: 92.5\%$100 \%$ ) consistent variant detection results compared with the orthogonal method, demonstrating HapICE's ability to accurately detect functional PSVs arising from gene conversion events. Through HapICE reanalysis, a diagnostic rate of 83.0\% (39/47) was achieved in this SDS high-risk cohort.

Moreover, HapICE was able to determine the phasing of the PSV haplotypes (in cis/trans) through proband-only analysis, while parental validation was necessary for Sanger sequencing to confirm compound heterozygous variants. Among the 31 diagnosable patients who had both heterozygous PSVs, 24 were available for further parental Sanger validation. HapICE haplotype results showed that the two functional PSVs were all in trans, which is consistent with the parental Sanger sequencing.

\subsection{Comparison between the HapICE result and conventional NGS analysis}

Since all of the variant detection results of HapICE were confirmed by Sanger sequencing, we then compared the HapICE result with conventional NGS analysis among the high-risk SDS cohort and evaluated their variant detection performances.

In conventional NGS analysis, $26(55.3 \%)$ samples had a potential molecular diagnosis, including 10 with a homozygous c. $258+2 \mathrm{~T}>\mathrm{C}$ variant and 16 harboring both heterozygous functional PSVs. Another 21 individuals were identified as carriers, including three with a heterozygous c.183_184delinsCT variant and 18 with a heterozygous c. $258+2 \mathrm{~T}>\mathrm{C}$ variant (Table $\mathbf{1}$ ).

\subsubsection{Diagnosable result comparison}

(1) Consistent diagnosis determined by HapICE and conventional NGS analysis All 26 patients with a diagnosable result by conventional NGS analysis were fully covered by HapICE diagnosable patients. However, only $18(69.2 \%)$ patients had consistent genetic variant conclusions by both methods, including 2 who had a homozygous c. $258+2 \mathrm{~T}>\mathrm{C}$ variant and 16 who harbored both heterozygous functional PSVs (Table 1).

\section{(2) Inaccurate pathogenesis diagnosis by conventional NGS analysis}

Apart from the consistent diagnosis cases, the molecular pathogenesis of 8 samples $(30.8 \%, 8 / 26)$ was inaccurately reported by conventional NGS analysis. Specifically, two individuals were mistakenly called the $S B D S$ homozygous c. $258+2 \mathrm{~T}>\mathrm{C}$ variant but were compound heterozygous at c.183_184delinsCT and c.258+2T $>\mathrm{C}$. The other six individuals were validated to simultaneously have the homozygous c. $258+2 \mathrm{~T}>\mathrm{C}$ and heterozygous c.183_184delinsCT variants, while conventional NGS analysis only detected the homozygous c. $258+2 \mathrm{~T}>\mathrm{C}$ variant.

\section{(3) False-negative missed by conventional NGS analysis}

False-negative results were also detected by conventional NGS analysis during SDS molecular diagnosis. For the validated diagnosable samples, 13 individuals with both heterozygous c.183_184delinsCT and c.258+2T $>\mathrm{C}$ variants were missed by 
conventional NGS analysis. These false-negative cases accounted for $41.9 \%$ (13/31) of all diagnosable individuals with both heterozygous functional PSVs, improving the diagnostic rate by $27.7 \%$ (13/47) through HapICE compared with conventional NGS analysis. All 13 cases identified only a heterozygous c. $258+2 \mathrm{~T}>\mathrm{C}$ variant through conventional analysis. A further manual review revealed that the interference resulted from the c.141C $>\mathrm{T}$ and/or c. $201 \mathrm{~A}>\mathrm{G}$ nonfunctional PSVs. When these two nonfunctional PSVs came with c.183_184delinsCT in cis, sequencing reads covering both variants were incorrectly mapped to $S B D S P 1$ in conventional analysis and thus missed c.183_184delinsCT (Figure 2C and D).

These comparison results showed that when both variants appeared on the two functional loci of $S B D S, 56.8 \%(21 / 37)$ of the c.183_184delinsCT variant was missed by conventional NGS analysis. In contrast, HapICE reanalysis could result in an improved diagnostic rate of $33.3 \%$ (13/39) and a more precise pathogenesis conclusion rate of $30.8 \%(8 / 26)$ compared with conventional NGS analysis, demonstrating its application potential in the clinical molecular diagnosis scenario.

\subsubsection{Other patients and available parental samples analysis result comparison}

We further investigated the variant detection results of HapICE and conventional NGS analysis at the two functional PSVs among the remaining 8 undiagnosed patients and 64 available parental samples of the cohort. Variant detection conclusions of the two methods were compared based on Sanger sequencing.

For these 72 individuals, HapICE showed that 64 and 8 were carriers and wild-type, respectively, while 51 carriers and 21 wild-type individuals were detected by conventional NGS analysis. Sanger sequencing was again 100\% (95\% CI: 95.0\%-100\%) consistent with HapICE, while conventional NGS analysis made incorrect variant calling in 17 (23.6\%) samples (Table 1).

\section{(1) Consistently identified carrier and wild-type individuals}

Altogether, 55 individuals were consistently identified at these two functional PSVs by HapICE and conventional NGS analysis, including 49 carriers (6 patients and 43 parental samples) and 6 wild-type individuals (all parental samples).

(2) False-positive and false-negative carriers detected by conventional NGS analysis

The inconsistently identified individuals reflected the false-positive and falsenegative carrier callings of conventional NGS analysis at the c.183_184 and c.258 loci. Specifically, among 17 samples with inconsistent results, two were false-positively called a heterozygous c. $258+2 \mathrm{~T}>\mathrm{C}$ variant by conventional NGS analysis. However, both HapICE and Sanger sequencing showed wild-type $S B D S$ and HapICE reported in cis n.484G $>$ A and n.466_467delinsTA in SBDSP1. These results showed that when both variants appeared on n.466_467 and n.484 of the SBDSP1 gene, conventional NGS analysis would prefer to call a false-positive c. $258+2 \mathrm{~T}>\mathrm{C}$ variant on $S B D S$. The other 15 parents were false-negatively identified as wild-type by conventional NGS analysis, while validated to include one with heterozygous c. $258+2 \mathrm{~T}>\mathrm{C}$, nine with heterozygous c.183_184delinsCT, and five with two functional PSVs in cis. 
The overall comparison of variant detection of the two functional PSVs on SBDS between HapICE and conventional NGS analysis showed that HapICE achieved an improved diagnostic rate of $27.7 \%$ in the SDS high-risk cohort $(\mathrm{n}=47)$ and a consistent variant detection rate of $100 \%$ (95\% CI: 96.7-100\%) among all validated individuals $(\mathrm{n}=111)$. Conventional NGS analysis only showed a consistent rate of $65.8 \%$ (95\% CI: $56.2 \%-74.5 \%$ ), resulting in both false-negative and false-positive conclusions in SDS molecular pathogenesis analysis.

\section{Discussion}

Shwachman-Diamond syndrome, primarily resulting from parental/pseudogene $(S B D S / S B D S P 1)$ conversion events, is a representative issue in NGS-based molecular diagnosis scenarios. Generally, when SDS is highly suspected based on typical clinical features, PCR sequencing with specific primer pairs can be applied to detect variants selectively within the $S B D S$ locus. However, the disease is thought to be underdiagnosed because of ambiguous clinical presentations ${ }^{[12]}$. In a clinical scenario where patients' disease-related phenotypes are mild and atypical, especially during infancy, exome sequencing that can screen for the entire gene set is commonly applied in pathophysiology examinations. Thus, it is of extreme importance to accurately detect pathogenic $S B D S$ variants resulting from gene conversion based on regular exome sequencing data. However, to our knowledge, few attempts have been made to provide a corresponding solution in previous studies, let alone systematic evaluation of the variant detection errors standard analysis would make, yet these works are essential for a better understanding of SDS.

In this study, we presented a novel tool, HapICE, to infer the haplotype consisting of PSVs for molecular diagnosis and carrier screening through conventional NGS data. We applied HapICE for reanalysis of two SBDS functional PSVs in an SDS high-risk cohort involving 47 Chinese pediatric patients. HapICE achieved a completely consistent result with Sanger sequencing and reached a diagnostic rate of $83.0 \%$. Compared with conventional NGS analysis, HapICE showed an improved diagnostic rate of $27.7 \%$ and an amended variant calling rate of $17.0 \%$ in this cohort. In addition to precise variant detection, HapICE could identify whether two heterozygous functional PSVs were in cis or trans by inferring the haplotype blocks from probandonly data. These results highlight HapICE as an efficient assay for the diagnosis of SDS in place of conventional NGS analysis.

Moreover, we systematically analyzed the variant detection features of c. $258+2$ and c.183_184 through conventional NGS analysis. We found that conventional NGS analysis for $S B D S$ gene exon 2 functional PSV callings would be frequently influenced by $S B D S P 1$ or other $S B D S$ nonfunctional PSVs, resulting in $25.2 \%$ false-negative and $1.8 \%$ false-positive variant detections, which may worsen the SDS underdiagnosis situation. The complexities of pathological diagnosis and the indispensable treatment of SDS patients highlight the need for efficient HapICE to be adopted in a large NGS diagnosis and carrier-testing platform to complement routine exome sequencing data analysis. 
In summary, as exome sequencing has become a preferred tool for molecular diagnosis, it is critical to involve gene conversion events that affect highly homologous regions such as $S B D S / S B D S P 1$ for more reliable variant detection. Although long-read sequencing technologies can largely overcome the inaccurate alignment problem of homologous reads, these platforms still either generate many sequencing errors or are too costly to be implemented in diagnostic laboratories. In this study, we showed that conventional NGS analysis can lead to the incomplete delineation of molecular pathogenesis and described the extent of the mistakes it will make in analyzing the two common functional PSVs of SBDS. We proposed a novel tool to solve the difficulty based on conventional short-read NGS data, which showed fantastic variant detection performance in an SDS cohort. Moreover, we applied HapICE to our in-house spinal muscular atrophy (SMA, MIM\#253300) patients to detect the functional PSV of SMN1 c.840C $>$ T that results from highly homologous SMN1 and SMN2 gene conversion events ${ }^{[5]}$. The results were confirmed by Sanger/multiplex ligation-dependent probe amplification (MLPA) (data not shown), demonstrating HapICE's potential to be further optimized and promoted to a wider range of clinical scenarios.

However, there are still some limitations in this study that can be further improved. The present study only focused on the two most common variants in the SBDS gene exon 2, and other pathogenic variants were not thoroughly explored. In addition, previous studies reported that structural variation (SV) in $S B D S$ was associated with SDS. Structural alterations, such as large deletions, duplications, insertions, or inversions, are anticipated due to the peculiar genomic architecture of the SBDS/SBDSP1 loci ${ }^{[32]}$. When the detected SNVs are insufficient to draw diagnosable conclusions in highly suspected clinical patients, the presence of SVs should be considered. However, HapICE did not address these situations and mainly focused on functional PSVs, which should be further improved for comprehensive variant analysis.

\section{Disclosure}

The authors declare no conflict of interest.

\section{Data availability statement}

The data that support the findings of this study are either included in the article (or in its supplementary files) or available from the corresponding author on reasonable request. The data are not publicly available due to privacy or ethical restrictions.

\section{Acknowledgment}

We thank the patients and their families, and referring physicians for their contribution to this study. We would like to thank Mr. W.B. Sheng for providing mathematical algorithm suggestions. This work was funded by the National Key Research and Development Program (2018YFC0116903), Shanghai Municipal Science and 
medRxiv preprint doi: https://doi.org/10.1101/2021.08.22.21262444; this version posted August 26, 2021. The copyright holder for this preprint (which was not certified by peer review) is the author/funder, who has granted medRxiv a license to display the preprint in

All rights reserved. No reuse allowed without permission.

Technology Major Project (2017SHZDZX01), the fellowship of China Postdoctoral Science Foundation (2020M681176), Shanghai Key Laboratory of Birth Defects (13DZ2260600), Shanghai Municipal Science and Technology Major Project (20Z11900600) and Clinical Skill and Innovation program of Shanghai Shenkang Hospital Development Center (SHDC12020CR6028-002).

\section{Author Contributions}

Conceptualization: Y.L., X.D., B.L., and W.Z.; Data curation: X.P., W.L., F.X., and L.Y.; Formal analysis: B.L., X.P., Y.L., X.D., and B.W.; Software \& Visualization: X.D., B.L., and Y.L.; Validation experiments: G.L.; Writing-original draft: B.L., X.P.; Writingreview \& editing: X.D., Y.L., Y.W., and H.W. All authors read and approved the final manuscript. The authors wish it to be known that, in their opinion, X.P. and X.D. should be regarded as joint First Authors.

\section{Ethics Declaration}

This study was approved by the ethics committees of Children's Hospital of Fudan University (2015-130). Counseling was performed by physicians before testing. Informed consent was obtained from the parents of each patient.

\section{Reference}

[1] Dong X R, Liu B, Yang L, Wang H J, Wu B B, Liu R C, Chen H B, Chen X, Yu S, Chen B, Wang S J, Xu X, Zhou W H, Lu Y L. Clinical exome sequencing as the first-tier test for diagnosing developmental disorders covering both CNV and SNV: a Chinese cohort [J]. J Med Genet, 2020, 57(8): 558-566.

[2] Stark Z, Tan T Y, Chong B, Brett G R, Yap P, Walsh M, Yeung A, Peters H, Mordaunt D, Cowie S, Amor D J, Savarirayan R, McGillivray G, Downie L, Ekert P G, Theda C, James P A, Yaplito-Lee J, Ryan M M, Leventer R J, Creed E, Macciocca I, Bell K M, Oshlack A, Sadedin S, Georgeson P, Anderson C, Thorne N, Gaff C, White S M, Alliance M G H. A prospective evaluation of wholeexome sequencing as a first-tier molecular test in infants with suspected monogenic disorders []]. Genet Med, 2016, 18(11): 1090-1096.

[3] Lionel A C, Costain G, Monfared N, Walker S, Reuter M S, Hosseini S M, Thiruvahindrapuram B, Merico D, Jobling R, Nalpathamkalam T, Pellecchia G, Sung W W L, Wang Z Z, Bikangaga P, Boelman C, Carter M T, Cordeiro D, Cytrynbaum C, Dell S D, Dhir P, Dowling J J, Heon E, Hewson S, Hiraki L, Inbar-Feigenberg M, Klatt R, Kronick J, Laxer R M, Licht C, MacDonald H, MercimekAndrews S, Mendoza-Londono R, Piscione T, Schneider R, Schulze A, Silverman E, Siriwardena K, Snead O C, Sondheimer N, Sutherland J, Vincent A, Wasserman J D, Weksberg R, Shuman C, Carew C, Szego M J, Hayeems R Z, Basran R, Stavropoulos D J, Ray P N, Bowdin S, Meyn M S, Cohn R D, Scherer S W, Marshall C R. Improved diagnostic yield compared with targeted gene sequencing panels suggests a role for whole-genome sequencing as a first-tier genetic test []]. Genet Med, 
medRxiv preprint doi: https://doi.org/10.1101/2021.08.22.21262444; this version posted August 26, 2021. The copyright holder for this preprint (which was not certified by peer review) is the author/funder, who has granted medRxiv a license to display the preprint in All rights reserved. No reuse allowed without permission.

2018, 20(4): 435-443.

[4] Srivastava S, Love-Nichols J A, Dies K A, Ledbetter D H, Martin C L, Chung W K, Firth H V, Frazier T, Hansen R L, Prock L, Brunner H, Hoang N, Scherer S W, Sahin M, Miller D T, Grp N E S R W. Meta-analysis and multidisciplinary consensus statement: exome sequencing is a first-tier clinical diagnostic test for individuals with neurodevelopmental disorders (vol 21, 2019, 2020) [J]. Genet Med, 2020, 22(10): 1731-1732.

[5] Chen J M, Cooper D N, Chuzhanova N, Ferec C, Patrinos G P. Gene conversion: mechanisms, evolution and human disease []]. Nat Rev Genet, 2007, 8(10): 762-775.

[6] Chen X, Wan L, Wang W, Xi W J, Yang A G, Wang T. Re-recognition of pseudogenes: From molecular to clinical applications []]. Theranostics, 2020, 10(4): 1479-1499.

[7] Pei B K, Sisu C, Frankish A, Howald C, Habegger L, Mu X J, Harte R, Balasubramanian S, Tanzer A, Diekhans M, Reymond A, Hubbard T J, Harrow J, Gerstein M B. The GENCODE pseudogene resource []]. Genome Biol, 2012, 13(9):

[8] Ebbert M T W, Jensen T D, Jansen-West K, Sens J P, Reddy J S, Ridge P G, Kauwe J S K, Belzil V, Pregent L, Carrasquillo M M, Keene D, Larson E, Crane P, Asmann Y W, Ertekin-Taner N, Younkin S G, Ross O A, Rademakers R, Petrucelli L, Fryer J D. Systematic analysis of dark and camouflaged genes reveals disease-relevant genes hiding in plain sight []]. Genome Biol, 2019, 20(

[9] Mandelker D, Schmidt R J, Ankala A, Gibson K M, Bowser M, Sharma H, Duffy E, Hegde M, Santani A, Lebo M, Funke B. Navigating highly homologous genes in a molecular diagnostic setting: a resource for clinical next-generation sequencing []]. Genet Med, 2016, 18(12): 1282-1289.

[10] Zhang Z L, Carriero N, Zheng D Y, Karro J, Harrison P M, Gerstein M. PseudoPipe: an automated pseudogene identification pipeline []]. Bioinformatics, 2006, 22(12): 1437-1439.

[11] Toiviainen-Salo S, Durie P R, Numminen K, Heikkila P, Marttinen E, Savilahti E, Makitie O. The natural history of Shwachman-Diamond syndrome-associated liver disease from childhood to adulthood []]. J Pediatr, 2009, 155(6): 807-811 e802.

[12] Farooqui S M, Ward R, Aziz M. Shwachman-Diamond Syndrome [M]. StatPearls. Treasure Island (FL). 2021.

[13] Cipolli M, Tridello G, Micheletto A, Perobelli S, Pintani E, Cesaro S, Maserati E, Nicolis E, Danesino C, Italian Registry O. Normative growth charts for Shwachman-Diamond syndrome from Italian cohort of 0-8 years old []]. BM] Open, 2019, 9(1): e022617.

[14] Szabo C E, Man O I, Serban R S, Kiss E, Lazar C F. Bruising as the first sign of exocrine pancreatic insufficiency in infancy []]. Med Pharm Rep, 2019, 92(2): 200-204.

[15] Tan S, Kermasson L, Hoslin A, Jaako P, Faille A, Acevedo-Arozena A, Lengline E, Ranta D, Poiree M, Fenneteau O, Ducou le Pointe H, Fumagalli S, Beaupain B, Nitschke P, Bole-Feysot C, de Villartay J P, Bellanne-Chantelot C, Donadieu J, Kannengiesser C, Warren A J, Revy P. EFL1 mutations impair elF6 release to cause Shwachman-Diamond syndrome [J]. Blood, 2019, 134(3): 277-290.

[16] Boocock G R B, Morrison J A, Popovic M, Richards N, Ellis L, Durie P R, Rommens J M. Mutations in SBDS are associated with Shwachman-Diamond syndrome [J]. Nat Genet, 2003, 33(1): 97-101.

[17] Leslie Steele J M R, Tracy Stockley, Berivan Baskin, Peter N Ray. De Novo Mutations Causing Shwachman-Diamond Syndrome and a Founder Mutation in SBDS in the French Canadian Population [J]. Journal of Investigative Genomics, 2014, 1(2): 00008.

[18] Woloszynek J R, Rothbaum R J, Rawls A S, Minx P J, Wilson R K, Mason P J, Bessler M, Link D 
medRxiv preprint doi: https://doi.org/10.1101/2021.08.22.21262444; this version posted August 26, 2021. The copyright holder for this preprint (which was not certified by peer review) is the author/funder, who has granted medRxiv a license to display the preprint in

All rights reserved. No reuse allowed without permission.

C. Mutations of the SBDS gene are present in most patients with Shwachman-Diamond syndrome []]. Blood, 2004, 104(12): 3588-3590.

[19] Nicolis E, Bonizzato A, Assael B M, Cipolli M. Identification of novel mutations in patients with Shwachman-Diamond syndrome []]. Hum Mutat, 2005, 25(4): 410.

[20] Bezzerri V, Cipolli M. Shwachman-Diamond Syndrome: Molecular Mechanisms and Current Perspectives [J]. Mol Diagn Ther, 2019, 23(2): 281-290.

[21] Watson C M, Dean P, Camm N, Bates J, Carr I M, Gardiner C A, Bonthron D T. Long-read nanopore sequencing resolves a TMEM231 gene conversion event causing Meckel-Gruber syndrome []]. Hum Mutat, 2020, 41(2): 525-531.

[22] Yamada M, Uehara T, Suzuki H, Takenouchi T, Inui A, Ikemiyagi M, Kamimaki I, Kosaki K. Shortfall of exome analysis for diagnosis of Shwachman-Diamond syndrome: Mismapping due to the pseudogene SBDSP1 []]. Am J Med Genet A, 2020, 182(7): 1631-1636.

[23] Liu B, Lu Y, Wu B, Yang L, Liu R, Wang H, Dong X, Li G, Qin Q, Zhou W. Survival Motor Neuron Gene Copy Number Analysis by Exome Sequencing: Assisting Spinal Muscular Atrophy Diagnosis and Carrier Screening []]. J Mol Diagn, 2020, 22(5): 619-628.

[24] Bean L J H, Funke B, Carlston C M, Gannon J L, Kantarci S, Krock B L, Zhang S, Bayrak-Toydemir $P$, Committee $A L Q A$. Diagnostic gene sequencing panels: from design to report-a technical standard of the American College of Medical Genetics and Genomics (ACMG) [J]. Genet Med, 2020, 22(3): 453-461.

[25] Yang L, Kong Y, Dong X, Hu L, Lin Y, Chen X, Ni Q, Lu Y, Wu B, Wang H, Lu Q R, Zhou W. Clinical and genetic spectrum of a large cohort of children with epilepsy in China []]. Genet Med, 2019, 21(3): 564-571.

[26] Li H, Durbin R. Fast and accurate short read alignment with Burrows-Wheeler transform [J]. Bioinformatics, 2009, 25(14): 1754-1760.

[27] Li H, Handsaker B, Wysoker A, Fennell T, Ruan J, Homer N, Marth G, Abecasis G, Durbin R, Proc G P D. The Sequence Alignment/Map format and SAMtools []]. Bioinformatics, 2009, 25(16): 2078-2079.

[28] Edgar R C. MUSCLE: multiple sequence alignment with high accuracy and high throughput [J]. Nucleic Acids Res, 2004, 32(5): 1792-1797.

[29] Li H, Handsaker B, Wysoker A, Fennell T, Ruan J, Homer N, Marth G, Abecasis G, Durbin R, Genome Project Data Processing S. The Sequence Alignment/Map format and SAMtools []]. Bioinformatics, 2009, 25(16): 2078-2079.

[30] Kent W J. BLAT--the BLAST-like alignment tool []]. Genome Res, 2002, 12(4): 656-664.

[31] Dempster A P, Laird N M, Rubin D B. Maximum Likelihood from Incomplete Data via the EM Algorithm [J]. Journal of the Royal Statistical Society: Series B (Methodological), 1977, 39(1): 1-22. [32] McReynolds L J, Jones K, Teshome K, Kennedy A, Shimamura A, Giri N, Alter B P, Savage S A. Large Genomic Deletions in Shwachman-Diamond Syndrome []]. Blood, 2018, 132 ( 
medRxiv preprint doi: https://doi.org/10.1101/2021.08.22.21262444; this version posted August 26, 2021. The copyright holder for this preprint (which was not certified by peer review) is the author/funder, who has granted medRxiv a license to display the preprint in

All rights reserved. No reuse allowed without permission.

\section{Figure legend}

Figure 1. The outline of the study design. We described the potential pitfall of conventional NGS analysis in detecting the most common pathogenic SBDS variants and then proposed a novel solution, HapICE, to optimize the variant analysis. We then applied HapICE for retrospective analysis in an SDS high-risk cohort for molecular diagnosis and validated by Sanger sequencing. We further evaluated and compared variant detection performance between HapICE and conventional NGS analysis according to Sanger sequencing results.

Figure 2. Schema of gene conversion between $S B D S$ and its pseudogene $S B D S P 1$ and the NGS sequence alignment surrounding the functional PSVs. (A). There are only six PSRs (paralogous sequence regions) that are different between the exon 2 of $S B D S$ (reverse strand) and the SBDSPI (forward strand) gene. (B) The chromosome location, reference base, and transcript location of the six PSRs between $S B D S$ exon 2 and SBDSP1. Among them, only two functional SBDS PSVs (paralogous sequence variants), c.258+2T $>C$ and c.183_184delinsCT, would affect the protein-coding and are often used as the $S B D S / S B D S P 1$ gene conversion events' markers. (C) and (D) show the NGS sequence pileups of read pairs $(2 * 150)$ in a sample with two wild-type copies of $S B D S P 1$ and one copy of $S B D S$ exon 2 with a heterozygous c. $258+2 \mathrm{~T}>\mathrm{C}$ variant and another $S B D S$ allele with both heterozygous c.183_184delinsCT and c. $201 \mathrm{~A}>\mathrm{G}$ variants confirmed by trio Sanger sequencing. On the $S B D S$ locus $(\mathbf{C})$, the c.183_184delinsCT and the c.201A $>\mathrm{G}$ variants were missed due to the ambiguously mapped reads. Meanwhile, the false-positive variants $n .424 \mathrm{~T}>\mathrm{C}$ and $n .533+10 \mathrm{C}>\mathrm{T}$ were called on the SBDSP1 locus (D).

Figure 3: A novel computational algorithm HapICE for SBDS/SBDSP1 gene conversion analysis using next-generation sequencing data. HapICE involves four main steps for $S B D S / S B D S P 1$ gene conversion analysis. Step1: Prepare the genespecific combined reference. Genome sequences from parental and pseudogene are aligned and marked into target region (where bases in parental gene/pseudogene genes were different, often PSVs) and other region (where bases were consistent). Step2: Align reads to the combined reference, and generate reads-to-region mapping content dataset. Sequencing reads are aligned to the combined reference and the exact base information located at the target region is recorded to generate the dataset. This dataset describes the mapping observation and is used to estimate haplotypes with probabilities. Step3: Haplotype inference. A pruning and recursive strategy is used to reduce calculation complexity and for each $\mathrm{k}(\mathrm{k}>1)$, the haplotype state space is augmented from $\mathrm{k}-1$, and the haplotypes with the highest probabilities inferred from the Expectation-Maximization (EM) step are reserved for the next step. For the EM step for $\mathrm{k}$ target region, the probability for each candidate haplotype was initialized, expected (E-Step) and maximized (M-Step) until convergence. Step4: Result visualization. Three visualization functions are provided to show the haplotype structures with probabilities, detailed recombination events, and supportive reads information. 
Table 1. Comparison of HapICE and conventional NGS data analysis for SBDS exon2 functional PSVs validated by Sanger sequencing.

Sanger sequencing

\begin{tabular}{|c|c|c|c|c|c|}
\hline \multicolumn{6}{|c|}{ Validated 39 diagnosed patients } \\
\hline \multirow{4}{*}{ HapICE } & & c. $258+2 \mathrm{~T}>\mathrm{C}, \mathrm{Hom}$ & \multicolumn{2}{|c|}{$\begin{array}{l}\text { c.183_184delinsCT, Het; } \\
\text { c. } 258+2 \mathrm{~T}>\mathrm{C}, \text { Het }\end{array}$} & $\begin{array}{l}\text { c.183_184delinsCT, Het; } \\
\text { c. } 258+2 \mathrm{~T}>\mathrm{C}, \text { Hom }\end{array}$ \\
\hline & c. $[258+2 \mathrm{~T}>\mathrm{C}] ;[258+2 \mathrm{~T}>\mathrm{C}]$ & 2 & \multicolumn{2}{|c|}{0} & 0 \\
\hline & c. $\left[183 \_184 d\right.$ delinsCT $] ;[258+2 \mathrm{~T}>\mathrm{C}]$ & 0 & \multicolumn{2}{|r|}{31} & 0 \\
\hline & c. $\left[183 \_184 d\right.$ delins CT;258+2T>C];[258+2T>C] & 0 & \multicolumn{2}{|r|}{0} & 6 \\
\hline \multirow{3}{*}{$\begin{array}{c}\text { Conventional } \\
\text { NGS }\end{array}$} & c. $258+2 \mathrm{~T}>\mathrm{C}$, Hom & 2 & \multicolumn{2}{|r|}{$2^{\mathrm{I}}$} & $6^{\mathrm{I}}$ \\
\hline & c.183_184delinsCT, Het; c.258+2T >C, Het & 0 & \multicolumn{2}{|r|}{16} & 0 \\
\hline & c. $258+2 \mathrm{~T}>\mathrm{C}, \mathrm{Het}$ & 0 & \multicolumn{2}{|r|}{$13^{\mathrm{II}}$} & 0 \\
\hline \multicolumn{6}{|c|}{ Validated other 72 wild-types and carries } \\
\hline \multirow{5}{*}{ HapICE } & & wild-type & $\begin{array}{c}\text { c. } 258+2 \mathrm{~T}>\mathrm{C}, \\
\text { Het }\end{array}$ & $\begin{array}{c}\text { c.183_184delinsCT } \\
\text {, Het }\end{array}$ & $\begin{array}{l}\text { c. } 183 \_184 \text { delinsCT, Het; } \\
\text { c. } 258+2 \mathrm{~T}>\mathrm{C} \text {, Het }\end{array}$ \\
\hline & wild-type & 8 & 0 & 0 & 0 \\
\hline & c. $258+2 \mathrm{~T}>\mathrm{C}, \mathrm{Het}$ & 0 & 33 & 0 & 0 \\
\hline & c.183_184delinsCT, Het & 0 & 0 & 26 & 0 \\
\hline & c. $\left[183 \_184\right.$ delinsCT;258+2T>C], Het & 0 & 0 & 0 & 5 \\
\hline Conventional & wild-type & 6 & $1^{\mathrm{III}}$ & $9^{\mathrm{III}}$ & $5^{\mathrm{III}}$ \\
\hline NGS & c. $258+2 \mathrm{~T}>\mathrm{C}, \mathrm{Het}$ & $2^{\mathrm{IV}}$ & 32 & 0 & 0 \\
\hline
\end{tabular}


c.183_184delinsCT, Het

Diagnostic rate $(n=47)$

HapICE

Conventional NGS
83.0\% (95\%CI: 69.2\%-92.4\%)

$55.3 \%$ (95\%CI: 40.1\%-69.8\%)
Overall consistent variant rate $(n=111)$

100\% (95\%CI: 96.7\%-100.0\%)

$65.8 \%$ (95\%CI: $56.2 \%-74.5 \%)$

Het: heterozygous, Hom: homozygous. Figures with superscript were inconsistent variants detected by conventional NGS analysis compared to the Sanger sequencing result. Specifically, "I" representing the inaccurate pathogenesis diagnoses, "II" were false-negative diagnosable SDS patients, "III" were false-negative SDS carriers, and "IV" were false-positive SDS carriers of conventional NGS analysis. 


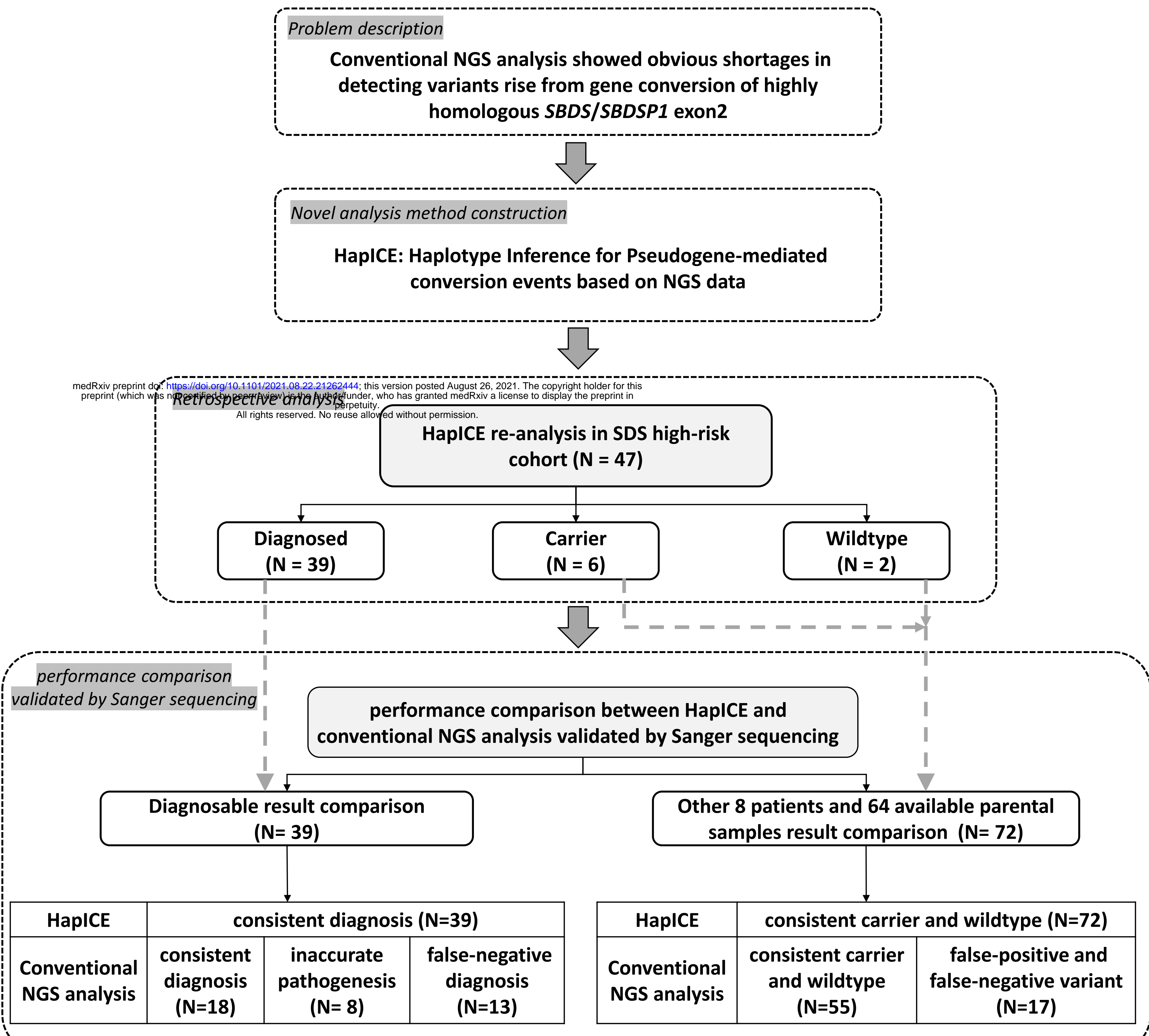



A medRxiv preprint doi: https://doi.org/10.1101/2021.08.22.21262444; this version pestedchughtst 26,2021 . The copyright holder for this
preprint (which was not certified by peer review) is the author/funder, who has granted medR SBDS (-): chr7:66452690-66460588

perpetuity.
All rights reserved. No reuse allowed without rmission.

$66,460,588$

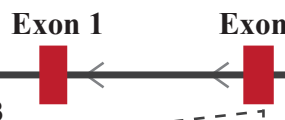

Exon 4

Exon 5
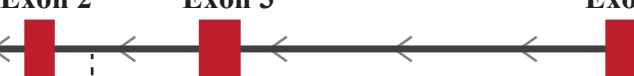

$66,452,690$

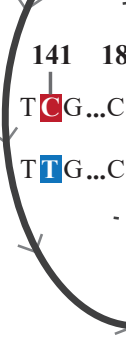

183184

$\begin{array}{ll}41 & 183+184 \\ C\end{array}$

$258+124$

AA GGAA GAT C T CAT

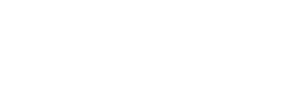

$258+126$

$72,299,952$

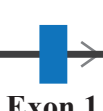

Exon 1
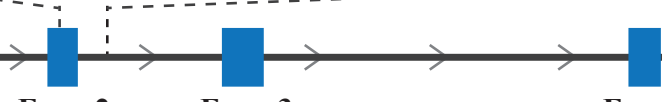

Exon 4

$72,307,978$

SBDSP1 (+): chr7:72299952-72307978

B

\begin{tabular}{|c|c|c|c|c|c|c|}
\hline PSR & PSR1 & PSR2 & PSR3 & PSR4 & PSR5 & PSR6 \\
\hline NM_016038 & c. 141 & c. $183 \_184$ & c. 201 & c. $258+2$ & c. $258+124$ & c. $258+126$ \\
\hline SBDS (-) & $66,459,316-C$ & $66,459,273-T A$ & $66,459,256-A$ & $66,459,197-T$ & $66,459,075-G$ & $66,459,073-G$ \\
\hline NR_001588 & n. 424 & n. $466 \_467$ & n. 484 & n. 533+10 & n. 533+132 & n. 533+134 \\
\hline SBDSP1 (+) & $\mathbf{7 2 , 3 0 1 , 2 8 4 - T}$ & $\mathbf{7 2 , 3 0 1 , 3 2 6 - C T}$ & $\mathbf{7 2 , 3 0 1 , 3 4 4 - G}$ & $\mathbf{7 2 , 3 0 1 , 4 0 3 - C}$ & $\mathbf{7 2 , 3 0 1 , 5 2 5 - A}$ & $\mathbf{7 2 , 3 0 1 , 5 2 7 - A}$ \\
\hline
\end{tabular}

C

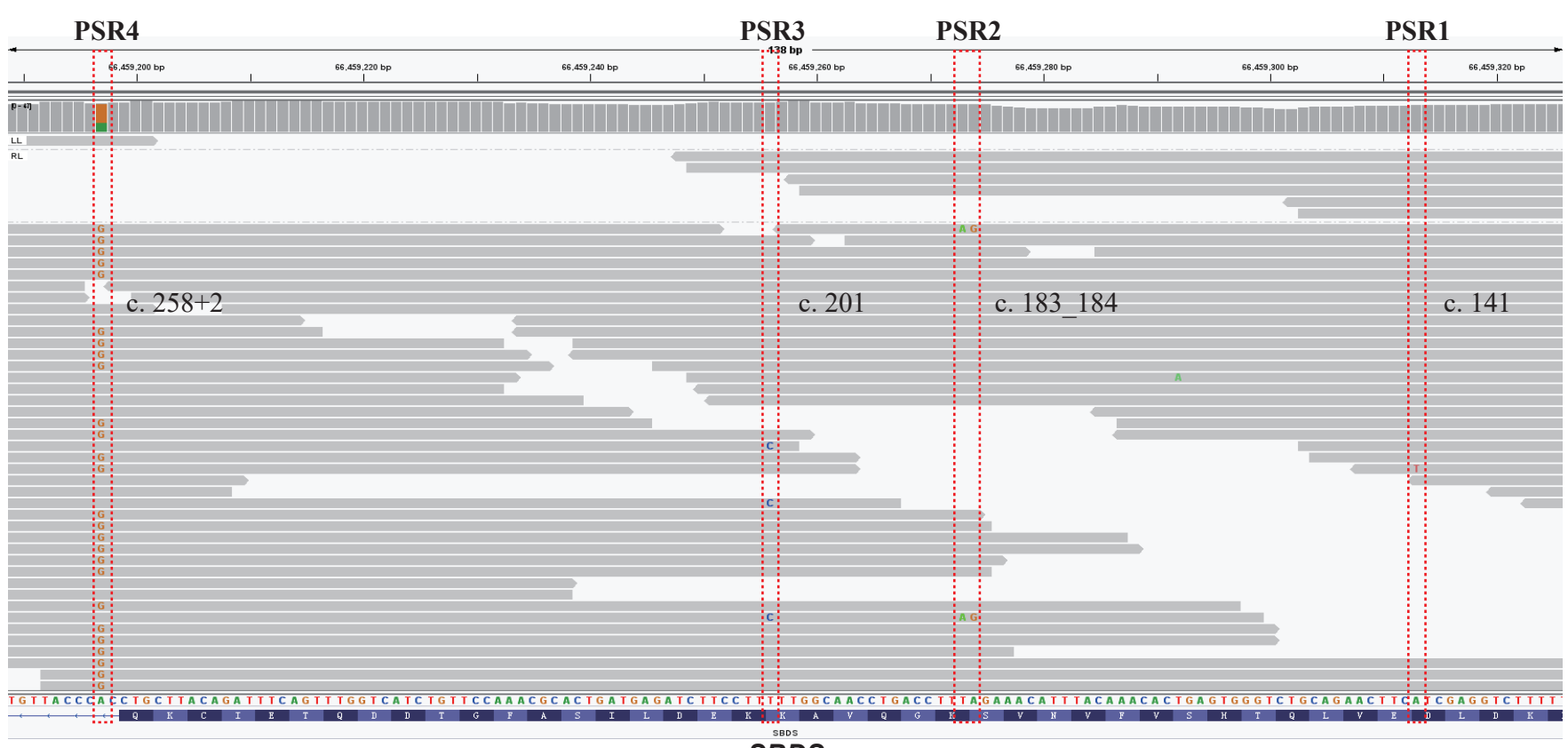

D

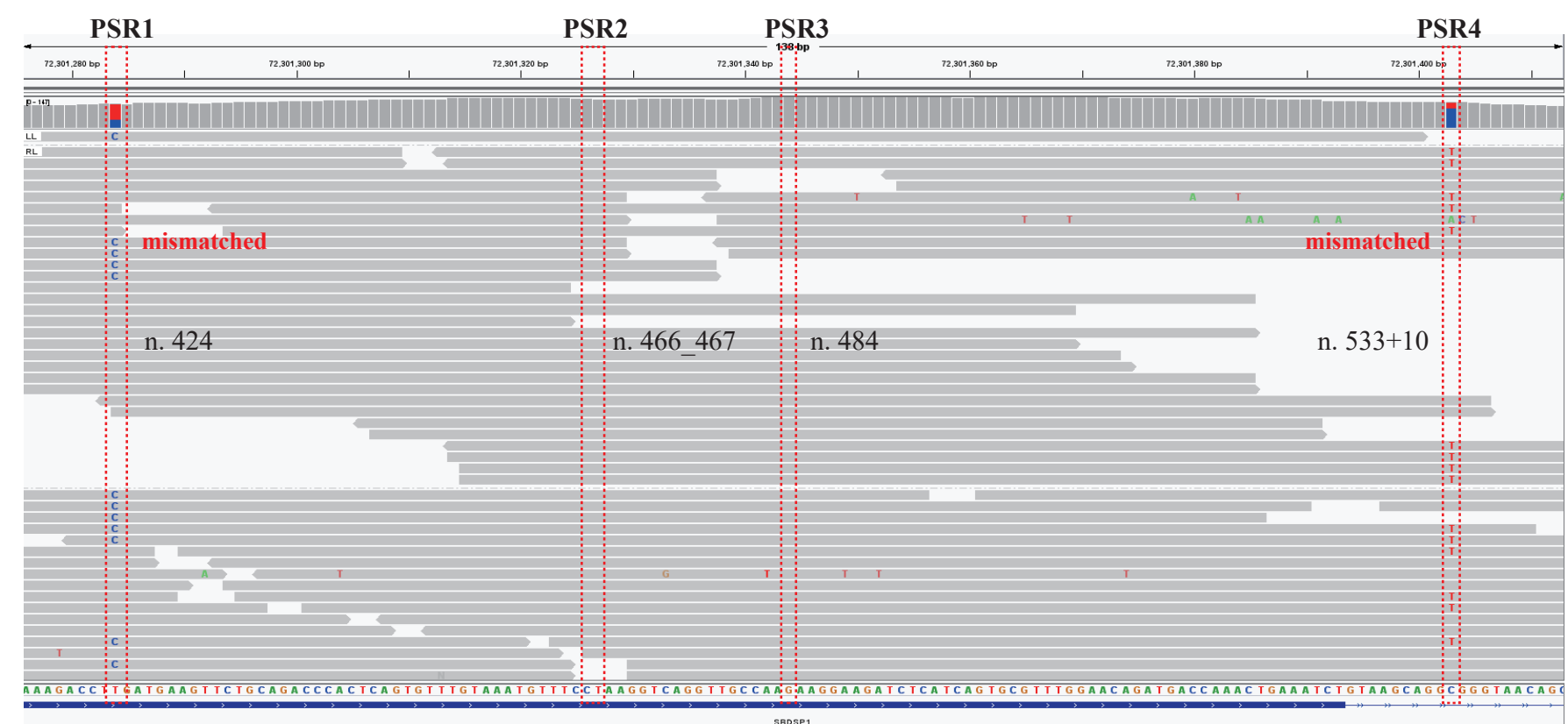




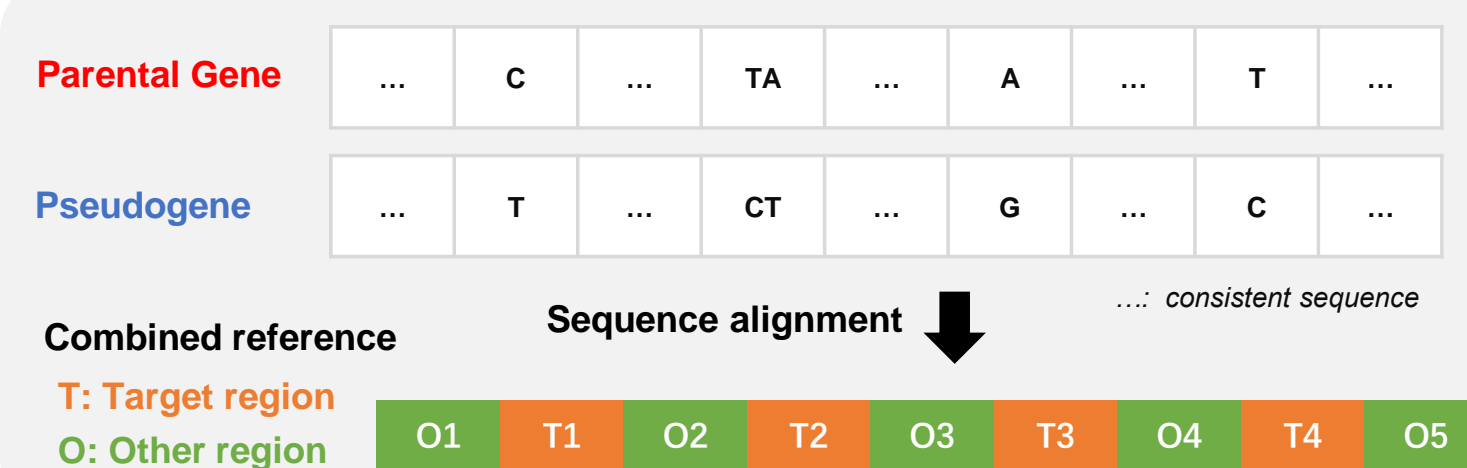

Step2: Align Sample reads to the combined reference and generate reads-to-region mapping content dataset

Combined reference
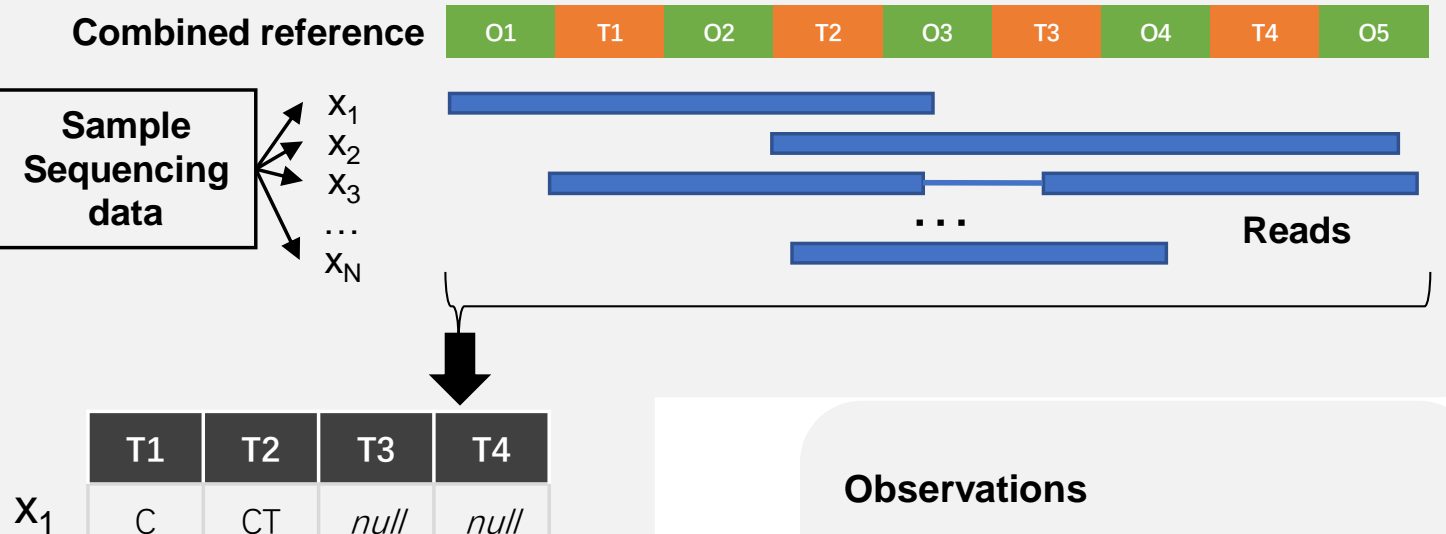

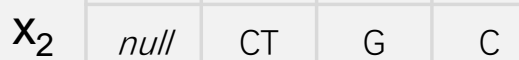

\begin{tabular}{l|l|l|l|l}
$\mathbf{X}_{3}$ & $\mathrm{C}$ & $\mathrm{TA}$ & $\mathrm{A}$ & $\mathrm{T}$
\end{tabular}

...

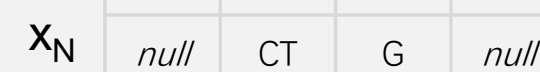

Reads-to-region mapping content dataset

\section{Observations}

- N reads: $x_{n}$

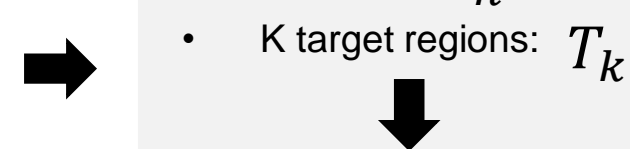

Purpose: Estimate haplotype

- M possible haplotypes: $h_{m}$ With haplotype probability: $\theta_{m}$
- Reduce calculation complexity by pruning and recursive strategy

$\boldsymbol{k}=\mathbf{1}$ Trivial estimation

$\boldsymbol{k}>\mathbf{1}$ Augment haplotype state space from $k-1$ to $k$

$\longrightarrow$ Do haplotype inference

Remain top haplotypes with highest probability

$\boldsymbol{k}=\boldsymbol{K} \quad$ Output haplotypes with probability

- Haplotype inference by ExpectationMaximization (EM) for $k$ target regions

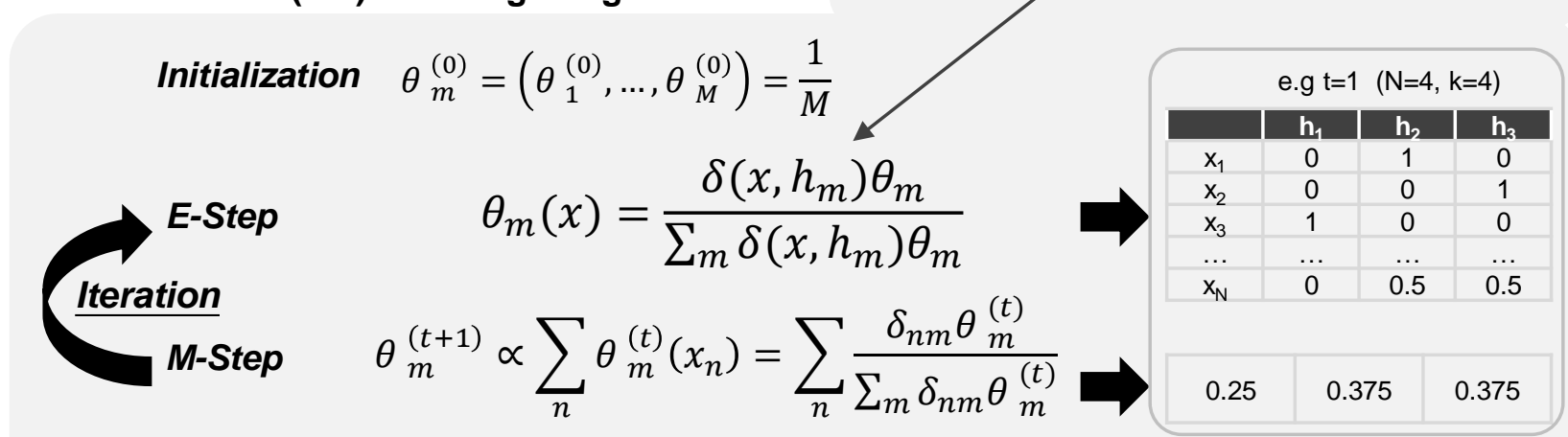

If Convergence, stop iteration

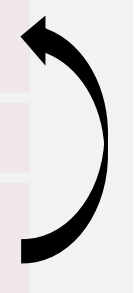

$\delta(x, h)$ indicate whether a certain read $x$ can support a haplotype $h$
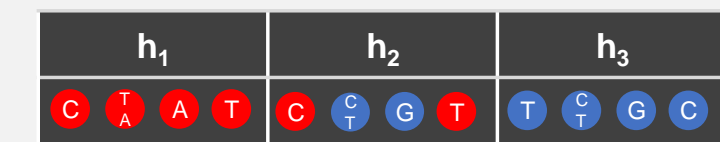

\begin{tabular}{l|l|l|l}
\hline $\mathrm{X}_{1}$ & $\delta_{1,1}=0$ & $\delta_{1,2}=1$ & $\delta_{1,3}=0$
\end{tabular}

\begin{tabular}{l|l|l|l}
$\mathrm{X}_{2}$ & $\delta_{2,1}=0$ & $\delta_{2,2}=0$ & $\delta_{2,3}=1$
\end{tabular}

\begin{tabular}{l|l|l|l}
$\mathbf{X}_{3}$ & $\delta_{3,1}=1$ & $\delta_{3,2}=0$ & $\delta_{3,3}=0$
\end{tabular}

\begin{tabular}{c|c|c|c}
$\cdots$ & $\cdots$ & $\cdots$ & $\cdots$ \\
\hline $\mathbf{X}_{\mathrm{N}}$ & $\delta_{N, 1}=0$ & $\delta_{N, 2}=1$ & $\delta_{N, N}=1$ \\
\hline
\end{tabular}

Step4: Result visualization

Proportion of gene recombination Haplotype supportive events between neighboring targets reads information

Inferred haplotypes of parental gene/pseudogene pairs

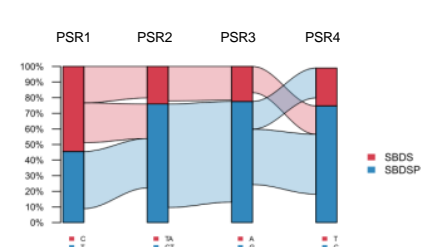

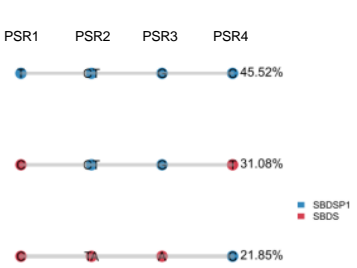

